\title{
SMOKING
}

\section{Is nicotine replacement therapy for smoking cessation effective in the "real world"? Findings from a prospective multinational cohort study}

\author{
Robert West, Xiaolei Zhou
}

Thorax 2007;62:998-1002. doi: 10.1136/thx.2007.078758

See end of article for authors' affiliations

\section{Correspondence to:} Dr Robert West, University College London, 2-6 Torrington Place, London WCIE 6BT, UK; robert. west@ucl.ac.uk

Received 26 January 2007 Accepted 2 May 2007

Published Online First 21 June 2007
Background: Increasing smoking cessation rates is an important goal in preventing lung cancer and chronic obstructive pulmonary disease. Nicotine replacement therapy (NRT) has been found in clinical trials to improve the chances of success at stopping, but recent cross-sectional survey data have raised doubts as to whether it is effective when used by smokers making quit attempts unsupervised outside clinical trials. Because of biases inherent in cross-sectional surveys, this issue can only be adequately addressed using longitudinal studies. This paper reports the first study of its kind to examine the issue.

Methods: The ATTEMPT cohort is a multinational cohort study with data collection by the internet which recruited smokers of $\geqslant 5$ cigarettes per day aged $35-65$ years who were intending to stop smoking within the next 3 months. Phase 1 began in spring 2003 and involved 2009 smokers from the USA, UK, Canada and France. Phase 2 involved 3645 smokers and included the same countries plus Spain. Follow-up assessments were carried out every 3 months. 492 smokers who made a quit attempt without formal behavioural support or bupropion in the first 3-month follow-up period were identified from phase 1, 357 of whom were followed up for a further 6 months. The phase 2 sample involved 906 smokers making quit attempts, 732 of whom were followed up. At baseline, demographic characteristics, smoking history and nicotine dependence were assessed. Smokers who made quit attempts were questioned on methods used to aid them. The main outcome measure was self-report of complete abstinence throughout both the 3-month periods following the quit date. Results: $35.6 \%$ of smokers followed up in phase 1 and $29.6 \%$ of those followed up in phase 2 used NRT. The odds ratios comparing abstinence for 6 months in those using and those not using NRT, adjusting for nicotine dependence, were $3.0(95 \% \mathrm{Cl} 1.2$ to 7.5$)$ for the phase 1 sample and $2.1(95 \% \mathrm{Cl} 1.0$ to 4.1$)$ for the phase 2 sample. The difference in success rates between those using NRT and those not using it, adjusted for the Fagerstrom test for nicotine dependence (FTND) score, was $6 \%$ in the phase 1 sample and $3.7 \%$ in the phase 2 sample. The improved odds of success were not explicable in terms of motivation to use some form of aid to cessation or differential loss to follow-up.

Conclusion: NRT use by smokers making self-initiated quit attempts without formal behavioural support is associated with improved long-term abstinence rates. l ncreasing smoking cessation rates is an important goal in preventing lung cancer and chronic obstructive pulmonary disease, ${ }^{1}$ but the chances of success of any given quit attempt are typically very low at less than $5 \% .^{2}$ More than 100 double blind, randomised, placebo controlled trials have been conducted evaluating nicotine replacement therapy (NRT) in the form of nicotine gum, transdermal patch or other products, ${ }^{3}$ and a meta-analysis of these trials found an average effect of NRT on the ability to remain abstinent for at least 6 months (minimum duration of follow-up required by Cochrane) following a quit attempt of 7 percentage points. ${ }^{3}$ There have been clinical trials of nicotine patches in an "over-the-counter" context, and these have also shown the efficacy of NRT. ${ }^{4}$ Longer term follow-up indicates that this size of effect translates into between $3 \%$ and $4 \%$ of smokers achieving abstinence for at least 8 years. $^{5}$ Although this is a modest effect, the cost of a treatment episode is low and the benefits of cessation are so great that NRT has been identified as one of the most costeffective life-preserving interventions available to medical science. ${ }^{6}$

An issue has been raised about the effectiveness of NRT outside clinical trials. Many smokers in the "real world" may use the products suboptimally leading to a lower level of effectiveness. In one large cross-sectional survey in California it was reported that smokers who said they had attempted to stop with the aid of NRT were no more likely to have abstained for 6 months or more than those who had not. ${ }^{7}$ However, this design could not test the hypothesis effectively. This is partly because it is difficult to control adequately for a range of potential confounders. ${ }^{8}$ Of particular importance is nicotine dependence which needs to be measured before the quit attempt is made rather than retrospectively several months later; more dependent smokers would be expected to be more likely to use NRT and less likely to succeed at stopping. In addition, this kind of study does not take into account the forgetting of failed quit attempts. The same team of researchers had already shown that forgetting was a potential source of bias $^{9}$ that could reduce or eliminate the apparent difference between quit attempts using effective methods and those that do not. Quit attempts made using less effective methods will fail more often but the failures will be forgotten so, the longer ago the quit attempt, the higher the apparent success rates overall and the greater convergence between rates associated with more effective and less effective methods. An indication of the extent of bias created is shown by the Californian study which yielded estimates of 6-month continuous abstinence rates in unaided quit attempts in excess of $20 \%$; this is more

Abbreviations: FTND, Fagerstrom test for nicotine dependence; NRT, nicotine replacement therapy 
than four times the rates found in prospective studies and is wholly unrealistic. Thus, cross-sectional surveys cannot answer questions of long-term effectiveness; longitudinal studies with frequent follow-up are required.

While there are a number of prospective and cross-sectional studies involving NRT in the literature, ${ }^{10-15}$ none of them directly address the crucial question of the difference in success rates between those using NRT and those not using it when they attempt to quit spontaneously outside a clinical trial setting without formal behavioural support.

This issue is of major public health importance because an estimated 2 million smokers used NRT in 2005 in the UK alone. ${ }^{16}$ An evaluation of a programme in New York to give away free nicotine patches to callers to a toll-free helpline found significantly higher short-term abstinence rates than in comparable smokers who had called the helpline before the scheme was introduced. ${ }^{14}{ }^{15}$ If these rates translate into improved long-term abstinence, this approach could prevent large numbers of premature deaths very cheaply but, if they do not, it just represents a waste of public resources.

This paper reports findings from a multinational cohort study that examined prospectively the 6-month continuous abstinence rates in a population sample of smokers making selfinitiated quit attempts with and without NRT, controlling for degree of nicotine dependence while smoking. NRT was available for purchase over-the-counter without prescription in all countries examined. During the period that the data were collected, smokers in the UK could also get partial or full reimbursement for NRT if they obtained a prescription from a doctor.

\section{METHODS}

The ATTEMPT cohort study is a multinational longitudinal cohort study carried out using the internet with 3-monthly assessments of cigarette smokers who at enrolment smoked $\geqslant 5$ cigarettes per day, were aged 35-65 years and were intending to quit within the next 3 months. Phase 1 of the study was initiated in the spring of 2003 in Canada, France, the UK and the USA with a sample of 2009 smokers. In phase 2 a second sample of 3645 smokers was added from the same countries plus Spain in 2004. Full details of the ATTEMPT methodology for phase 1 can be found elsewhere. ${ }^{17}$ The methodology for phase 2 was similar. The study was designed to examine a range of issues concerning smoking cessation including the short and medium term health effects of stopping and factors associated with success or otherwise of quit attempts.

At the first post-baseline wave for each sample (3 months after enrolment), smokers were asked: "During the past 3 months (90 days), have you made a serious attempt to stop smoking cigarettes for good that lasted for at least a day (24 hours)? Yes/No". They were also asked to indicate from a list which of a range of methods they had used in that attempt. Among these methods were the various NRT devices (patch, gum, lozenge, sublingual tablet, inhaler and nasal spray), as well as items relating to behavioural support (formal behaviour modification programmes, counselling and help from a stop smoking clinic) and use of bupropion. In the phase l sample, 578 reported making a quit attempt in the 3 months leading up to the first follow-up. In the phase 2 sample the figure was 983 . Only these participants who made a quit attempt were used in our analyses. A total of 214 participants (37.0\%) in the phase 1 sample and $308(31.3 \%)$ in the phase 2 sample reported that they had used some form of NRT. We were interested in the effect of NRT in smokers not receiving any form of behavioural support and we also wished to disentangle any effect from an effect of bupropion, so we excluded those who reported that they had used some form of behavioural support or bupropion
( $N=86$ in the phase 1 sample of whom 44 used NRT; $N=77$ in the phase 2 sample of whom 40 used NRT). There were not enough of those using behavioural support or bupropion to perform a separate evaluation of their association with success at stopping smoking.

We also wished to assess the effect of motivation to use some form of support to address the question of whether any effect of NRT could be explained merely in these terms. We did this by determining those that had used any of hypnotherapy, acupuncture, herbal remedies, the internet and books into a single dichotomous variable. We chose these forms of support because using them can be presumed to reflect a level of motivation to stop smoking comparable with that of NRT users, but these methods can be presumed in general to be minimally effective. We did not include telephone helplines because these have been found in a recent review to have levels of effectiveness comparable to face-to-face behavioural support. ${ }^{18}$ Note that we were not able to assess the specific effectiveness of the various forms of support individually because of small numbers. The purpose of this analysis was only to assess the possible role of motivation to use some form of support with stopping; 113 of the phase 1 sample and 154 of the phase 2 sample used some other form of support.

At baseline (at the start of the cohort) data on age, sex, marital status, educational level and ethnic group were collected. We also recoded daily cigarette consumption and measured their nicotine dependence using the Fagerstrom test for nicotine dependence (FTND). ${ }^{19}$ Table 1 gives details of the two study samples.

A total of $357(72.6 \%)$ were followed up 3 months and 6 months later from the phase 1 sample; 127 used NRT and 230 did not; 60 used what was deemed likely to be an ineffective method of support and 297 did not. A total of 732 (80.8\%) were followed up from the phase 2 sample; 217 used NRT and 515 did not; 113 used what was deemed likely to be an ineffective method of support and 619 did not. Note that the categories of NRT use and use of "ineffective" support were not mutually exclusive. FTND data were missing for 3 smokers in phase 1 and 9 in phase 2, so the sample sizes for the logistic regression analyses were 354 for phase 1 and 723 for phase 2 . There were no differences between those successfully followed up and those lost to follow-up in either sample, except that those followed up in the phase 2 sample were slightly older.

At the survey points 3 and 6 months after the period in which the quit attempt was made, participants were asked whether they had been abstinent throughout the preceding 90 days. We designated as abstinent for 6 months those who reported that they had been abstinent for the full 90 days (without any lapses) at both follow-up visits. This was the primary outcome measure. Note that in principle those designated as abstinent for 6 months had been abstinent for at least 6 months, but it could have been up to 9 months depending on when they began their quit attempt relative to the first survey point.

\section{Statistical analysis}

The statistical power to detect a 7\% difference (the size of effect found in clinical trials ${ }^{3}$ ) between those using NRT and those not using NRT was $60 \%$ in the phase 1 sample and more than $80 \%$ in the phase 2 sample. Continuous variables were compared using $t$ tests and categorical variables using $\chi^{2}$ tests. For the primary analyses, logistic regression analyses were used to assess the effectiveness of NRT among subjects who made a quit attempt. A second logistic regression analysis was used to assess the association between the use of "ineffective" aids and success. 
Table 1 Characteristics of phase 1 and phase 2 samples (smokers who made a quit attempt without using behavioural support or bupropion)

\begin{tabular}{|c|c|c|c|}
\hline & Followed up & Not followed up & Total \\
\hline \multicolumn{4}{|l|}{ Phase 1 sample } \\
\hline Total (N) & 357 & 135 & 492 \\
\hline From USA (N) & 256 & 86 & 342 \\
\hline From Canada (N) & 44 & 16 & 60 \\
\hline From France (N) & 31 & 14 & 45 \\
\hline From UK (N) & 26 & 19 & 45 \\
\hline Mean (SD) age (years) & $48.6(8.1)$ & $48.7(8.7)$ & $48.6(8.3)$ \\
\hline Married, \% (N) & $52.4(187)$ & $48.9(66)$ & $51.4(253)$ \\
\hline Female, \% (N) & $45.1(161)$ & $48.1(65)$ & $45.9(226)$ \\
\hline White, \% $(\mathrm{N}) \dagger$ & $90.5(314)$ & $91.3(115)$ & $90.7(429)$ \\
\hline Post secondary education, \% (N) $\ddagger$ & 73.7 (263) & 68.7 (92) & $72.3(355)$ \\
\hline Reporting health as "poor", \% (N) & $9.5(34)$ & $8.9(12)$ & $9.3(46)$ \\
\hline \multicolumn{4}{|l|}{ Smoking, \% (N)§: } \\
\hline $5-10$ cigs/day & $16.0(57)$ & $17.0(23)$ & $16.3(80)$ \\
\hline $11-15$ cigs/day & $45.4(162)$ & $45.2(61)$ & $45.3(223)$ \\
\hline $16-20$ cigs/day & 34.4 (123) & $34.8(47)$ & $34.6(170)$ \\
\hline $21+$ cigs/day & $4.2(15)$ & $3.0(4)$ & 3.9 (19) \\
\hline Mean (SD) dependence: FTND & $5.0(2.3)$ & $5.1(2.5)$ & $5.0(2.4)$ \\
\hline Using NRT, \% (N) & 35.6 (127) & $31.9(135)$ & $34.6(170)$ \\
\hline Using other support, \% (N) & $16.8(60)$ & $24.4(33)$ & $18.9(93)$ \\
\hline \multicolumn{4}{|l|}{ Phase 2 sample } \\
\hline Total (N) & 732 & 174 & 906 \\
\hline From USA (N) & 162 & 16 & 178 \\
\hline From Canada (N) & 29 & 2 & 31 \\
\hline From France (N) & 259 & 60 & 319 \\
\hline From the UK (N) & 188 & 71 & 259 \\
\hline From Spain (N) & 94 & 25 & 119 \\
\hline Mean (SD) age (years)* & $46.1(7.5)$ & $44.7(7.1)$ & $45.8(7.4)$ \\
\hline Married, \% (N)** & 49.9 (364) & $48.3(84)$ & 49.6 (448) \\
\hline Female, \% (N) & $46.3(339)$ & $47.7(83)$ & $46.6(422)$ \\
\hline White, \% (N)+† & $95.0(678)$ & $94.6(158)$ & $94.9(836)$ \\
\hline Post secondary education, \% (N)‡ & 60.5 (443) & $55.5(96)$ & $59.6(539)$ \\
\hline Mean (SD) cigs per day§ & $18.0(9.9)$ & $18.2(9.8)$ & $18.0(9.9)$ \\
\hline Mean (SD) dependence: FTND $\ddagger \ddagger$ & $4.3(2.4)$ & $4.4(2.7)$ & $4.3(2.5)$ \\
\hline Using NRT, \% (N) & $29.6(217)$ & $29.3(51)$ & $29.6(268)$ \\
\hline Using other support, \% (N) & $15.4(113)$ & $13.2(23)$ & $15.0(136)$ \\
\hline \multicolumn{4}{|c|}{ 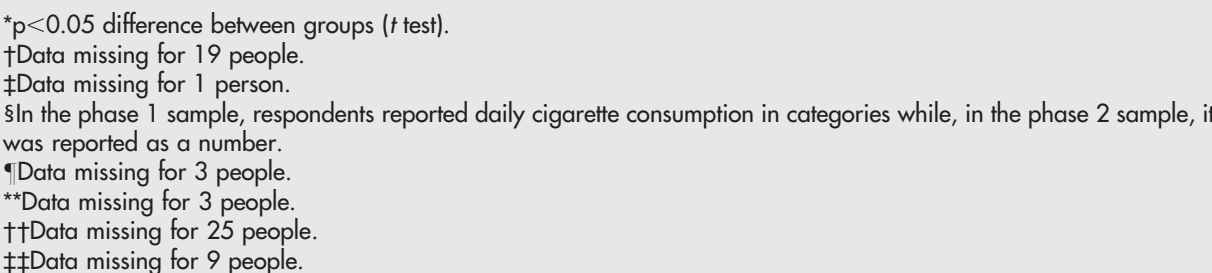 } \\
\hline
\end{tabular}

\section{RESULTS}

The mean (SD) FTND scores were higher in those using NRT than in those not using it: $5.4(2.3)$ vs $4.8(2.3)$ in the phase 1 sample ( $\mathrm{p}=0.02$, analysis of variance) and $4.8(2.3)$ vs $4.1(2.5)$ in the phase 2 sample $(\mathrm{p}<0.001)$. It was also somewhat higher in those who used other forms of support than in those who did not use them: $5.4(2.1)$ vs $5.0(2.0)$ in the phase 1 sample $(\mathrm{p}=\mathrm{NS})$ and $4.8(2.3)$ vs $4.2(2.5)$ in the phase 2 sample $(\mathrm{p}<0.02)$.
Table 2 shows the results of logistic regression analyses predicting 6-month continuous abstinence as a function of use of NRT and other support, adjusting for FTND. It is clear in both samples that the use of NRT was associated with an increase in success rates. In neither the phase 1 nor the phase 2 samples were educational level, age or sex significantly associated with NRT use. However, in the phase 2 sample but not in the phase 1 sample, NRT use was significantly associated with country $\left(\chi^{2}=22.7, p<0.001\right)$, so the logistic regression was repeated

Table 2 Results of logistic regression analyses to test associations between NRT use or use of other aids and continuous abstinence for 6 months

\begin{tabular}{|c|c|c|c|}
\hline & \multicolumn{3}{|c|}{ Odds ratio $(95 \% \mathrm{Cl})$ adjusting for FTND } \\
\hline & Phase 1 sample & Phase 2 sample & Combined sample \\
\hline Model 1 & & & \\
\hline $\begin{array}{l}\text { NRT vs no NRT } \\
\text { Model } 2\end{array}$ & $3.0^{*}(1.2$ to 7.5$)$ & $2.1 *(1.0$ to 4.1$)$ & $2.2^{*}(1.3$ to 3.9$)$ \\
\hline Other aid vs no other aid $†$ & $0.8(0.2$ to 3.1$)$ & 1.6 (0.7 to 3.7$)$ & 1.4 (0.7 to 2.7$)$ \\
\hline
\end{tabular}

NRT, nicotine replacement therapy; FTMD, Fagerstrom test of nicotine dependence.

${ }^{*} \mathrm{p}<0.05$ difference between groups (two-tailed).

†Other aids include hypnotherapy, acupuncture, herbal remedies, the internet or books. 
including both country and FTND as covariates. This did not alter the findings; the odds ratio remained $2.1 \quad(\mathrm{p}<0.05)$.

The adjusted difference in likelihood of achieving 6 months of abstinence between NRT users and non-users was $6.0 \%$ in the phase 1 sample and $3.7 \%$ in the phase 2 sample. In phase 1 the unadjusted difference was $5.9 \%(9.4 \%$ in the NRT group and $3.5 \%$ in those not using NRT). In phase 2 the unadjusted difference was $2.6 \%$ (6.9\% in the NRT groups compared with $4.3 \%$ in those not using NRT).

Combining the data from the two samples and including the phase as a covariate along with FTND, the odds of achieving 6 months of abstinence among those using NRT were $2.2(95 \%$ CI 1.3 to 3.9) times higher than those not using it $(\mathrm{p}<0.005)$. The adjusted difference in success rates (with phase and FTND as covariates) was $4.3 \%$. The unadjusted difference in success rates was $3.8 \%$ (7.8\% in the NRT group and $4.0 \%$ in those not using NRT).

There was no evidence that the use of forms of support that would not be expected to have specific efficacy but which could be presumed to signal a high level of motivation to quit was associated with an increase in success rates (table 2).

\section{DISCUSSION}

NRT use was associated with improved chances of long-term abstinence when controlling for nicotine dependence. The abstinence rates in those not using NRT were similar to estimates from untreated samples in clinical trials and other longitudinal studies. The size of effect is broadly what would be predicted from the clinical trials. The effect did not appear to be a function of motivation to use some form of support. Two strong features of this study are the fact that the finding was replicated in two separate samples and the frequent follow-up (every 3 months) which we believe is unique in population studies of this kind.

A potential problem with cohort studies is a bias caused by loss to follow-up. We showed that those lost to follow-up did not differ at baseline from those followed up. There was no differential loss to follow-up in those using NRT and those not using it, so this could not affect the findings. The fact that the sample was recruited by the internet is another obvious potential source of bias as internet users may be more likely to use NRT appropriately because they are more likely to have more education. In fact, the sample was very close in terms of demographic and smoking characteristics to samples drawn from household surveys, ${ }^{17}$ and while they did tend to have a higher educational level, there was no suggestion of an interaction between education level and NRT effectiveness in either sample. A third potential source of bias is reliance on self-reported quit rates. This may lead to an overestimation of successful quitting, but there is no reason why it should contribute to a difference in success rates as a function of NRT use compared with non-NRT use. Indeed, if there was greater motivation to report abstinence in those using NRT, one would also expect to see this with other forms of support and that is not what was observed. Fourthly, the sample was drawn from smokers expressing an intention to try to stop within the next 3 months, and it remains possible that NRT is less effective in smokers who make quit attempts without having formulated any intention to do so previously. ${ }^{20}$

The fact that the sample was limited to smokers of $\geqslant 5$ cigarettes per day means that it cannot address the potential effectiveness of NRT in very light smokers, but the product labels indicate that they are suitable for smokers of 10-15 or more cigarettes per day anyway and we do not have evidence from randomised trials of efficacy in light smokers.

The questionnaire did not permit matching NRT usage to individual quit attempts if respondents made more than one quit attempt in the 3-month window. Where smokers made more than one quit attempt, there may therefore have been some noise introduced into the data. However, this would, if anything, weaken any associations found.

There are many questions that we were not able to answer because of a lack of statistical power. We were not able to assess the effectiveness of bupropion or face-to-face behavioural support in addition to NRT, nor could we establish whether there was an interaction between NRT use and the country of residence of the smokers. Similarly, we were not able to determine whether NRT was more or less effective in smokers with different sociodemographic characteristics. These issues are all important but will require additional studies.

In conclusion, this paper contradicts findings from crosssectional surveys requiring recall of quit attempts over an extended period, and supports the findings from the clinical trial literature that NRT use is associated with a greater likelihood of remaining abstinent for at least 6 months in smokers making self-initiated quit attempts without additional behavioural support. This association does not appear to be explained by a greater commitment to stopping smoking.

\section{ACKNOWLEDGEMENTS}

The authors thank Sanofi-Aventis Recherche for making the data available for the analysis, Alicia Gilsenan and Elizabeth Sherrill from RTI International for their helpful comments on a draft and contribution to data collection, and Cancer Research UK for providing part funding for the paper.

\section{Authors' affiliations \\ Robert West, University College London, London, UK \\ Xiaolei Zhou, RTI Health Solutions, RTI, Research Triangle Park, North Carolina, USA}

Funding: Sanovi-Aventis made the data available for this paper and funded the ATTEMPT cohort study. Robert West's salary is mainly paid by Cancer Research UK. The research is independent of the funder.

Competing interests: Robert West has undertaken paid research and consultancy and has received hospitality from manufacturers of smoking cessation medications. He also has a share in a patent for a novel nicotine delivery device. Xiaolei Zhou is employed by RTI International which was paid by Sanofi-Aventis to implement the ATTEMPT cohort study.

Ethical approval was granted by RTI International's Institutional Review Board.

\section{REFERENCES}

1 Doll R, Peto R, Boreham J, et al. Mortality in relation to smoking: 50 years' observations on male British doctors. BMJ 2004;328:1519.

2 Hughes JR, Keely J, Naud S. Shape of the relapse curve and long-term abstinence among untreated smokers. Addiction 2004;99:29-38.

3 Silagy C, Lancaster T, Stead L, et al. Nicotine replacement therapy for smoking cessation. In: Cochrane Library, Issue 4. Oxford: Update Software, 2004.

4 Hughes JR, Shiffman S, Callas P, et al. A meta-analysis of the efficacy of over-thecounter nicotine replacement. Tob Control 2003;12:21-7.

5 Yudkin P, Hey K, Roberts S, et al. Abstinence from smoking eight years after participation in randomised controlled trial of nicotine patch. BMJ 2003;327:28-9

6 National Institute for Clinical Excellence. Nicotine replacement therapy (NRT) and bupropion for smoking cessation, National Institute for Clinical Excellence Technology Appraisal Guidance No.38. London: NICE, 2002.

7 Pierce JP, Gilpin EA. Impact of over-the-counter sales on effectiveness of pharmaceutical aids for smoking cessation. JAMA 2002;288:1260-4.

8 Stead LF, Davis RM, Fiore MC, et al. Effectiveness of over-the-counter nicotine replacement therapy. JAMA 2002;288:3109-10.

9 Gilpin E, Pierce JP. Measuring smoking cessation: problems with recall in the 1990 California Tobacco Survey. Cancer Epidemiol Biomarkers Prev 1994;3:613-7.

10 Zhu S, Melcer T, Sun J, et al. Smoking cessation with and without assistance: a population-based analysis. Public health benefit of over-the-counter nicotine medications. Am J Prev Med 2000;18:305-11.

11 Thorndike AN, Biener L, Rigotti NA. Effect on smoking cessation of switching nicotine replacement therapy to over-the-counter status. Am J Public Health 2002;92:437-42.

12 Alberg AJ, Stashefsky Margalit R, et al. The influence of offering free transdermal nicotine patches on quit rates in a local health department's smoking cessation program. Addict Behav 2004;29:1763-78. 
13 Hasford J, Fagerstrom KO, Haustein KO. A naturalistic cohort study on effectiveness, safety and usage pattern of an over-the-counter nicotine patch. Cohort study on smoking cessation. Eur J Clin Pharmacol 2003;59:443-7.

14 Miller N, Frieden TR, Liu SY, et al. Effectiveness of a large-scale distribution programme of free nicotine patches: a prospective evaluation. Lancet 2005;365:1849-54

15 Cummings KM, Hyland A, Fix B, et al. Free nicotine patch giveaway program 12-month follow-up of participants. Am J Prev Med 2006;31:181-4.

16 Taylor T, Lader D, Bryant A, et al. Smoking-related behaviour and attitudes, 2005. London: Office of National Statistics, 2006.
17 West R, Gilsenan A, Coste F, et al. The ATTEMPT cohort: a multi-national longitudinal study of predictors, patterns and consequences of smoking cessation; introduction and evaluation of internet recruitment and data collection methods. Addiction 2006;101:1352-61.

18 Stead LF, Lancaster T, Perera R. Telephone counselling for smoking cessation. In: Cochrane Library, Issue 1. Oxford: Update Software, 2006.

19 Heatherton TF, Kozlowski LT, Frecker RC, et al. The Fagerstrom Test for Nicotine Dependence: a revision of the Fagerstrom Tolerance Questionnaire. Br J Addict 1991;86:1119-27.

20 West R, Sohal T. "Catastrophic" pathways to smoking cessation: findings from national survey. BMJ 2006;332:458-60.

\section{PULMONARY PUZZLE}

\section{Bilateral pulmonary alveolar infiltrate and prostate tumour in a 54-year-old man}

\section{Clinical presentation}

A 54-year-old man presented with a 6-month history of cough, dyspnoea, weight loss and obstructive urinary symptoms. One week before admission to hospital he complained of urinary retention and left lumbar pain. The digital rectal examination was abnormal with a firm and significantly enlarged prostate. The serum prostate-specific antigen level was $0.05 \mathrm{ng} / \mathrm{ml}$ and the serum creatinine level was $1.9 \mathrm{mg} / \mathrm{dl}$. Ultrasonography and CT scanning of the pelvis showed a 150 g prostate tumour with invasion of the bladder and left ureteral meatus causing an uretero-hydronephrosis and enlargement of the pelvic lymph nodes (fig lA). Perihilar bilateral alveolar filling pattern was seen on the chest radiograph (fig lB) and a CT scan of the chest revealed a crazy paving pattern characterised by normal pulmonary areas superimposed on an area of ground-glass opacity and thickening of the interlobular septa (fig lC).

\section{Question}

What is the relation between the prostate tumour and the pulmonary disease?

See page 1018

This case was submitted by:

André A Figueiredo, Guilherme Brandão, Leonardo C Dentz, Christiane Meurer Alves, Antônio E S Stroppa Santa Casa de Misericórdia de Juiz de Fora and Núcleo Interdisciplinar de Pesquisa em Urologia (NIPU) da Universidade Federal de Juiz de Fora, Minas Gerais, Brazil

Correspondence to: Professor André A Figueiredo, Rua Irineu Marinho 365, Apto 801 - Bloco 3, Bom Pastor, Juiz de Fora MG, Brazil CEP 36021-580; andreavaresef@gmail.com

doi: $10.1136 /$ thx.2007.083717
A

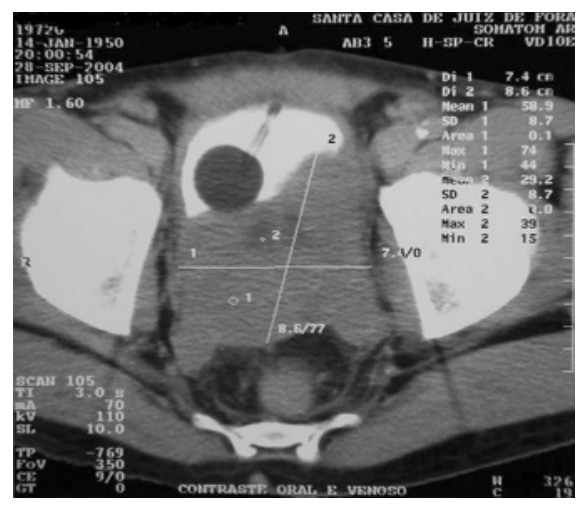

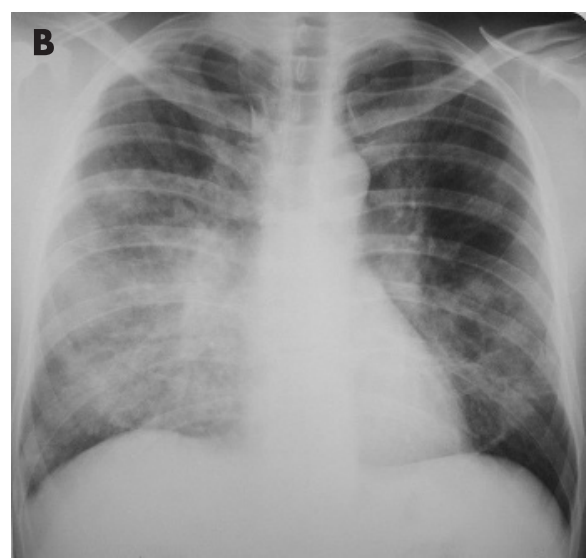

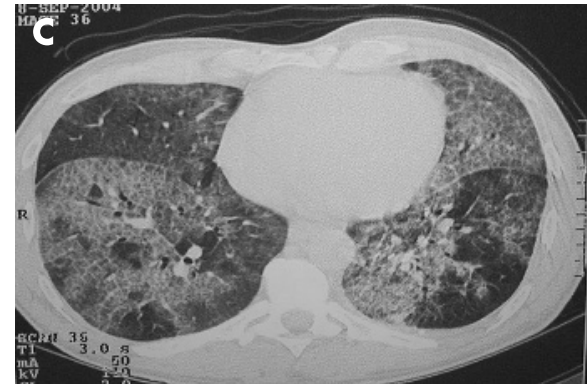

Figure 1 (A) CT scan of the pelvis showing a prostate tumour with bladder invasion. (B) Chest radiograph showing a bilateral perihilar alveolar infiltrate. (C) Chest CT scan showing ground-glass opacity. 